\title{
Approche des problématiques du texte d'écran
}

La création du sens et le lecteur

\section{Caroline Angé}

\section{CpenEdition}

\section{Journals}

Édition électronique

URL : http://journals.openedition.org/recherchestravaux/90

DOI : $10.4000 /$ recherchestravaux.90

ISSN : 1969-6434

Éditeur

UGA Éditions/Université Grenoble Alpes

Édition imprimée

Date de publication : 15 juin 2008

Pagination : 71-80

ISBN : 978-2-84310-125-0

ISSN : 0151-1874

Référence électronique

Caroline Angé, «Approche des problématiques du texte d'écran », Recherches \& Travaux [En ligne], 72 | 2008, mis en ligne le 15 décembre 2009, consulté le 03 février 2021. URL : http://

journals.openedition.org/recherchestravaux/90 ; DOI : https://doi.org/10.4000/recherchestravaux.90

(c) Recherches \& Travaux 
Caroline ANGÉ

Université Stendhal - Grenoble 3

\section{Approche des problématiques du texte d'écran : la création du sens et le lecteur}

Questionner la migration du texte de l'imprimé sur le support numérique comme un medium éditorial implique une prise en compte spécifique du contenu mis en ligne - fût-il le manuscrit - en tant qu'il constitue une forme particulière de texte. Mais ce contenu est lui-même intriqué dans le contexte spécifique du texte d'écran qui p-rocède à une mise en forme, c'est-à-dire à l'agencement formel de ce contenu. Ainsi, les acteurs traditionnels de la chaîne du livre voient l'émergence de nouveaux venus qui, tout en contribuant à l'existence du texte sur ce support, tendent à le transformer.

Mon propos développera quelques éléments de ce savoir méthodologique et technique de conception, en ce qu'il permet de mettre l'accent sur des questions étroitement liées aux contenus, cruciales du point de vue de la valeur ajoutée d'un produit interactif multimédia, et plus encore des enjeux culturels. L'intention qui préside à toute pratique de conception consiste en ce que la présence de la machine disparaisse (les contraintes de sa matérialité) et qu'il ne subsiste que le plaisir du texte. Là est le défi essentiel de ceux qui commandent l'utilisation de ces nouvelles machines textuelles : les strates de conception qui encadrent le texte visent à se fondre dans le produit fini.

L'objet de mon intervention est double : je convoquerai la pratique de conception multimédia d'un point de vue méthodologique d'une part, en mettant l'accent sur quelques-unes de ses étapes dans les solutions qu'elle propose, pour ouvrir, d'autre part, une réflexion sur le processus de construction du sens du point de vue du lecteur/utilisateur. 
Tenter de problématiser la relation au texte d'écran conduit à prendre la mesure de la complexité de ce dispositif qui, plus qu'une machine textuelle, est aussi un média destiné à la communication. Ce faisant, il propose d'autres usages du texte dans ses modalités spécifiques de production du sens. Le parti pris tient moins à l'exposé de préconisations qu'à l'énoncé de quelques questions, tout en m'appuyant ici et là sur des exemples puisés dans un corpus littéraire pour illustrer mon propos. Enfin, je conclurai sur la question de l'édition de fragments, en ce qu'elle est révélatrice d'enjeux contemporains liés au numérique, l'objet même des préoccupations de ce colloque.

L'ancrage théorique de cette réflexion est éclectique pour appréhender une textualité hétérogène et composite (sémiologie, linguistique et phénoménologie), mais trouve un cadrage dans une approche communicationnelle pragmatique, attendu qu'il s'agit de penser un nouveau contexte de diffusion et donc de communication de contenus. En effet, les pratiques d'édition électronique ne peuvent faire l'économie d'une réflexion sur le processus de signification du point de vue du lecteur, dans la mesure où il s'agit moins de lui permettre une simple navigation (sur le réseau) que de lui donner des outils d'appropriation de contenus littéraires. Chaque situation de communication interactive pose en des termes différents des questions qui se ressemblent: «Quelles sont les compétences attendues? Quelle liberté accorde-t-on à l'utilisateur? Quelle est la valeur ajoutée d'un produit interactif' ?» S'il n'y a de réponses que contextuelles, les questions méritent toutefois d'être posées.

\section{Méthodologie de conceptions : une prescription de questions}

L'intérêt des propositions de conception multimédia se profile dans la formulation : la prescription de questions. J'entends par là la prise de conscience du contexte de création de sens propre au dispositif numérique. En effet, s'il convient de s'appuyer sur des méthodes éprouvées dans la conceptionréalisation (notamment la scénographie), la spécificité de la conception multimédia se fonde sur une dimension évaluative et critique d'un véritable questionnement orienté vers la pratique de la lecture, c'est-à-dire la position de l'usager.

Là réside le vrai enjeu dans un parcours canonique de création-conception de contenus interactifs. Il ne s'agit pas simplement de transposer sur un autre support, mais de mettre en forme le texte qui sera donné à lire et à voir, en

I. C. Lacotte, «L'interactivité», cours en ligne, Université d'Avignon, 2003. Ce cours n'existe plus sous cette forme et n'est donc plus accessible. 
tenant compte de ses spécificités. Le texte est donc réélaboré par une écriture qui tend à maitriser son agencement formel et éditorial adapté à la situation d'interaction en ligne.

Après une description des étapes propres à tout projet, c'est-à-dire le contexte de communication (l'identité symbolique de l'émetteur, le public cible, les objectifs - informer, rechercher, publier - si l'on prend par exemple les axes d'un hypertexte de recherche), se profile une véritable écriture de mise en scène. Cette écriture minimale vise la mise en cohérence des différentes strates du texte d'écran, c'est-à-dire ce qui relève du discursif (l'énonciation qui va déterminer un horizon d'attente), le textuel au sens des mises en forme possibles (parcours, structure), et la dimension sensible, c'est-à-dire le traitement du contenu par les traits verbaux, sonores, et visuels, de l'écriture multimédia.

De cet agencement résulte une proposition qui coordonne les grands principes de la conception, à savoir l'«expérience utilisateur», les types d'actes de langage, une cohérence entre le type idéal du lecteur et la dimension symbolique (les valeurs de l'énonciateur). La phase de conception fonctionnelle doit servir à mettre en place une orientation générale de l'interface proposée, c'està-dire la promesse d'énonciation. Il s'agira de poser précisément la relation que l'on engage par la scénarisation d'une stratégie énonciative. Concrètement, cette étape se traduit par l'intégration d'éléments de médiation d'accès au texte, tel que le paratexte, dont l'enjeu pour le concepteur réside dans la coexistence harmonieuse de la visualité (désigne les signes outils : menus déroulants, barre de navigation) et de la visibilitée (c'est-à-dire des contenus lisibles).

Nous pouvons traduire cette promesse par l'énumération de quelques questions :

- Quelle est la valeur ajoutée de l'édition numérique par rapport à un support traditionnel, c'est-à-dire le livre-objet?

- Quelle est la source de valeur pour le lecteur? Que lui propose-t-on et comment? Autrement dit: par quelles fonctionnalités pratiques, par quelles médiations, accède-t-il au manuscrit?

- Quels outils de recherche et de scénographie convient-il de mettre en place?

- Quelles sont les compétences et plus encore les attentes qu'on attribue à l'utilisateur visé?

- Dans le cas de produits interactifs culturels, quels sont les niveaux d'expertise attendus?

2. F. Plégat-Soutjis, «Sémantique graphique des interfaces, représentations, valeurs et usages», Communication et Langages, $\mathrm{n}^{\circ} \mathrm{I} 42,2004$, p. I9-32. 
- Quels sont les types de parcours proposés, compte tenu des fonctions que l'on attribue au contenu (informatif, explicatif, incitatif, etc.)?

Ce qui nous amène à cette question essentielle : quel type de relation l'œuvre en ligne (l'accès à des manuscrits) instaure-t-elle avec son utilisateur? C'est dans la prescription de cette dernière question que se situe l'apport d'une démarche de conception orientée vers l'utilisateur. La rigidité des bases de données, notamment, génère un chemin d'accès balisé, alors qu'une interactivité navigationnelle permet la mise en place d'un univers métaphorique susceptible de rencontrer l'idéal-type de l'utilisateur profilé en associant des images à des actions possibles. Ces deux exemples soulignent qu'un système d'interaction très limité ne signifie pas nécessairement un échange simplifié.

Cette énumération de questions témoigne précisément du fait que les réponses ne sont que contextuelles, et dépendent non pas tant du dispositif pour lequel on opte - à la fois au sens du support et de l'outil (base de données, cédérom, site Internet, etc.) - que du contexte d'utilisation. C'est pourquoi, il ne s'agit pas tant de choisir d'éditer selon les fonctionnalités offertes par le numérique qu'en fonction de la pertinence du support par rapport à l'usage qui sera fait du contenu (lecture, recherche, publication). Dans le cas des manuscrits, il conviendra de voir s'il s'agit de notes de cours, de manuscrits, de textes non publiés. Le cas du site www.roland-barthes.com ${ }^{3}$ illustre un échec à travers lequel l'explication première, à savoir la rentabilité insuffisante du site, a peut-être aussi à voir avec la pertinence des usages.

La fonction ne suffit pas à orchestrer une relation. Face à cette difficulté, la pratique de conception met en place une stratégie énonciative, informative dans le cas où il s'agit de donner au lecteur les moyens de gérer fonctionnellement les contenus en ligne, mais aussi incitative, symbolique. À cette promesse d'énonciation répond la prévision en amont de programmes d'usages adaptés en fonction de parcours aisément identifiables par le lecteur. Si l'on prend l'exemple d'un hypertexte documentaire, la question se pose de savoir à quels lecteurs on s'adresse afin de proposer différents modes d'accès correspondant aux publics visés par le dispositif. Penser la mise en ligne de documents numérisés est corrélatif de propositions d'usage.

Définir les instances de réception que l'on convoque (lecteur explorateur, lecteur critique) permet une approche raisonnée de l'organisation des contenus interactifs et, partant, de leur organisation structurelle. On touche là à la question

3. Site consacré à l'œuvre de Barthes, fermé depuis novembre 2006. Sur ce point, nous renvoyons aux communications de $\mathrm{Cl}$. Coste et de G. Bellon, «Comment vivre ensemble de Roland Barthes : vie et mort d'un site littéraire» et «Le vivre et mourir en ligne du Vivre ensemble de Roland Barthes», dans le présent ouvrage, p. 20 I et 2 I 7 . 
de l'architecture du contenu soulevée par l'hypertextualisation. La mise en scène implique une structure logique qui pense l'inclusion du lecteur-utilisateur dans le dispositif. Pour reprendre le terme de Jean Clément ${ }^{4}$, il convient de mettre en place une «rhétorique hypertextuelle», afin que ce nouveau dispositif de publication donne les clés de sa propre discursivité. Cette forme de textualité qui offre au lecteur une collection de documents associés entre eux par des liens dynamiques rend possible la manipulation interactive des données. Du fait que les techniques de mise en forme qui ont fait du texte un instrument de savoir intellectuel, telles que l'unité physique, la totalité, l'achèvement, la continuité, n'ont plus cours, des outils de contextualisation permettront de concilier les enjeux cognitifs avec un lecteur désormais sujet d'action.

En nous attachant à l'ancienne page d'accueil du site HyperNietzsches, pionnier en matière d'édition électronique de manuscrits (fig. I), on repérera les usages prescrits par le biais des fonctionnalités de l'écriture.

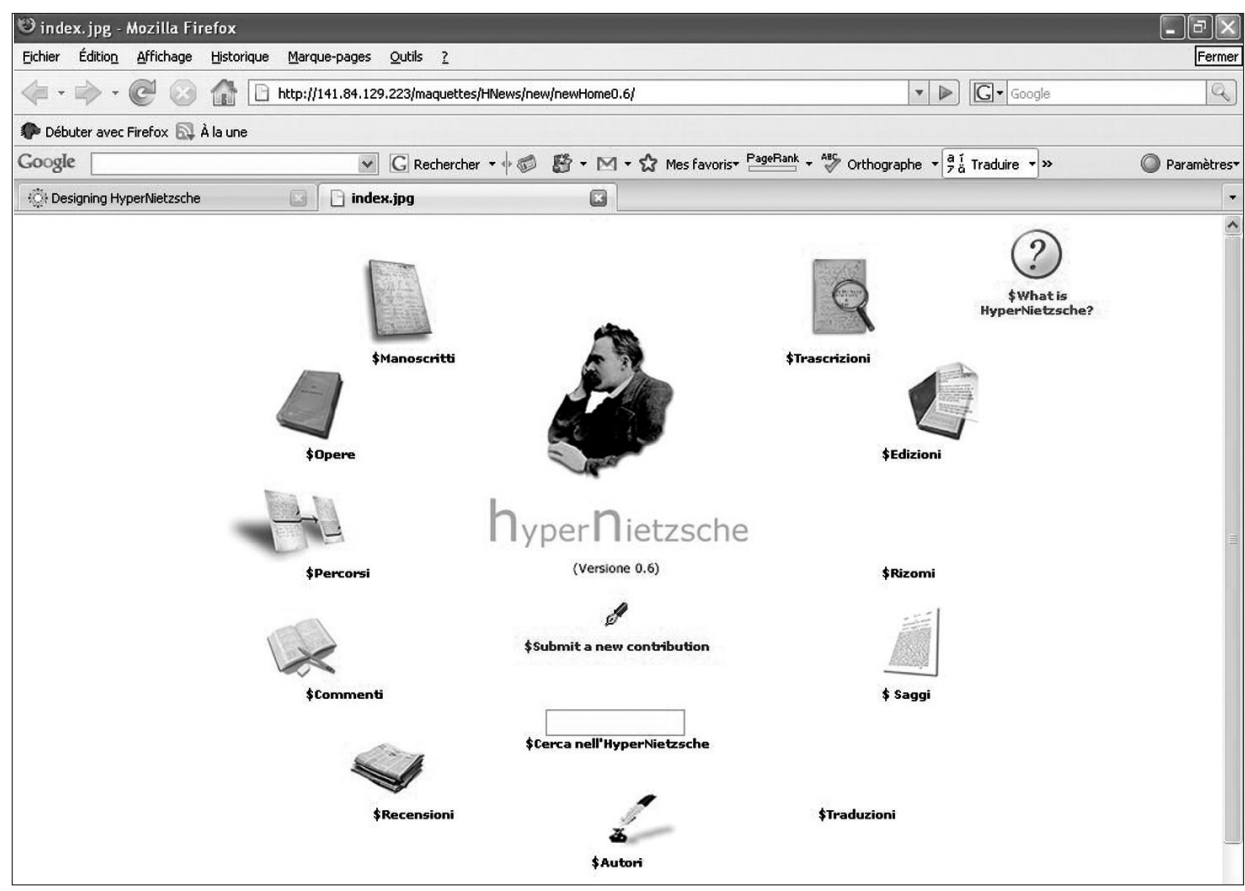

Fig. I. Ancienne page d'accueil du site HyperNietzsche (version o.6).

4. J. Clément, «Hypertexte et fiction : une affaire de liens», Les Défis de la publication sur le Web: hyperlectures, cybertextes et méta-éditions, coordonné par J.-M. Salaün et Ch. Vandendorpe, Villeurbanne, Presses de l'ENSSIB, 2004, p. 7I.

5. http://www.hypernietzsche.org. Le site est actuellement en cours de refonte pour une transition d'HyperNietzsche vers NietzscheSource. Il n'est donc pas accessible pour le moment. 
Cette page apparaît comme une présentation «sémiodiscursive» d'actions dans lesquelles l'utilisateur peut s'engager. Chaque accès (ou programme de consultation possible) est proposé à la fois par un signe linguistique (par exemple, "naviguer dans la connaissance, chercher des informations») et un signe iconique reprenant l'imaginaire livresque du chercheur. Cette coprésence n'a pas seulement une fonction d'ancrage mais vise à associer le faire (le geste) à l'acte de sélection du lien. C'est précisément la possibilité de ces différents parcours de lecture issus de divers objets textuels (carnets, feuillets, manuscrits) qui donne sens à la pratique. Les mots engendrent des actions qui permettent de contrôler les utilisations possibles de l'interface, tout en proposant des outils de recherche et de gestion des erreurs qui guideront le lecteurutilisateur. Cette mise en œuvre de parcours narratifs doit passer par la mise en scène de l'interface à travers la succession des pages écran, la hiérarchisation du contenu (son découpage et ses zones d'interaction) selon des critères d'organisation structurelle (position dans la page, surface, forme, etc.). Mettre l'accent sur la structure conduit à penser ses effets sur les modes de perception. La description phénoménologique de la perception met l'accent sur l'expérience immédiate et sensible qui lie, pour le dire trivialement, le dedans et le dehors. Le lecteur vise une zone spatiale : un acte qui rejoint une chose en soi (l'écran) à travers des états subjectifs non spatiaux, selon une gradation qui va d'une lecture de détail à une approche périphérique. Ce que nous apprennent ces différents degrés de perception plus ou moins conscients, c'est bien la nécessité de lier le vécu du sujet-lecteur à l'objet en orientant l'intensité sémiotique du message. Ce qui est donné à voir est étroitement corrélé à la conscience de l'impact visuel. Dans ce sens, la fragmentation des blocs textuels sur l'écran en constitue un exemple, en ce qu'elle permet une interpellation visuelle : ce quelque chose qui se détache sur le fond de la page oriente ma perception. Il convient donc de conférer «un corps sensible à l'information $^{6} »$ par des choix plastiques qui feront vivre des valeurs.

On voit là l'intérêt d'une méthodologie de conception qui conduise à mettre en place une dynamique éditoriale propre au contexte numérique dans la prise en compte d'une cohérence globale entre la dimension discursive, la structure, et la dimension sensible du texte d'écran. Mais, on l'aura compris, cette mise en perspective succincte montre que l'essentiel est davantage dans la formulation de questions sur les conditions de réception par le lecteur-utilisateur que dans «le faire technique» lui-même. La prescription d'usages oriente en produisant un cadre de contraintes et de possibles, mais ne saurait

6. N. Pignier, B. Drouillat, Penser le webdesign : modèles sémiotiques pour les projets multimédias, L'Harmattan, 2004, p. Io. 
en aucun cas préjuger de l'appropriation. Si les propriétés dynamiques du texte d'écran sont interactives, cela ne suffit pas à générer la construction d'une relation entre le contenu et le co-énonciateur, c'est-à-dire de l'interaction.

\section{Pacte énonciatif hypertextuel et construction du sens : les enjeux de la publication numérique}

Comment construire une expérience donnée en partage? Comment passer de la prescription des usages à la production du sens? S'il n'y a pas de réponses à ces questions (fort heureusement), leur formulation même nous oriente vers la valeur ajoutée propre à la publication numérique - qui tient moins au support qu'aux modalités de mises en relation avec le lecteur. Or, le texte d'écran implique une autre dynamique de construction de sens pour le lecteur, en ce que le dispositif transforme - c'est-à-dire «change de forme au sens propre du terme ${ }^{7} \gg-$ le contenu donné à lire. Pour approcher la dynamique hypertextuelle ${ }^{8}$ de production du sens, il convient de voir en quoi ce processus est réorganisé par rapport aux logiques énonciatives sur support imprimé.

La rencontre de l'informatique et du texte interroge le rôle que peut jouer le lecteur dans la construction du sens, et la façon dont il peut s'approprier le texte. Tout acte de lecture prend place dans un ensemble de conventions, références communes entre l'auteur et son lecteur. Reste à voir en quoi les clauses du contrat de lecture hypertextuelle instaurent une nouvelle dynamique de production de sens.

La lecture, plus qu'un acte d'interprétation, révèle le texte. Le geste acquiert un statut singulier: cliquer c'est lire. Le texte est désormais soumis aux manipulations du lecteur, qui se trouve en position de le faire exister.

La configuration hypertextuelle de l'écrit (sa structuration) confère au lecteur la maitrise de ce qu'il fait apparaitre, ce par quoi il constitue un texte nouveau à partir de ces assemblages. Par là, le lecteur voit ses points d'introduction dans le texte démultipliés. Dès lors, on comprendra que la structure du texte d'écran inverse le processus de création du sens. A une actualisation des significations se substitue la production d'un sens dans l'appropriation d'un champ textuel reconfigurable. La lecture hypertextuelle caractérisée par la navigation offre la possibilité de construire son propre cheminement intellectuel à partir des données qu'elle convoque. Certes, cela n'est pas nouveau : tout acte de

7. E. Souchier, «Lorsque les écrits de réseaux cristallisent la mémoire des outils, des médias et des pratiques», Les Défis de la publication sur le Web, op. cit., p. 93.

8. L'hypertexte est un système permettant de gérer une collection d'informations auxquelles on peut accéder de manière non séquentielle. 
lecture est un acte de transformation. Plus encore, le lire est indissociable de l'écrire. Cette instance d'énonciation fait basculer certaines prérogatives pragmatiques de l'énonciation vers le lecteur qui reçoit, traite, et actualise l'information. Mais la délégation conduit à un leurre : l'auteur s'efface tout en guidant le lecteur dans un scénario déjà écrit d'avance.

Dans ce sens, l'appropriation du sens passe par une renégociation constante du pacte lectoral entre ce qui a été écrit, dit, et organisé, et ce qui va se passer. Le processus de construction du sens s'intègre dans un contexte qui implique le lecteur comme instance cognitive et sujet social. Le concepteur aura donc à penser les termes de la relation qu'il veut générer dans son application, pour produire des formes d'interpellation qui donneront l'illusion d'une construction conjointe. De cette mise en situation découlent des choix narratifs et la promesse d'énonciation. Or, il ne suffit pas d'impliquer le lecteur pour produire du sens. Le problème est alors de «savoir où, dans l'univers infini des données $[\ldots]$ commence la sémiose, autrement dit à partir de quel moment, dans quelles conditions et comment une donnée quelconque d'un monde réel ou imaginaire - se transforme en signe ${ }^{9} »$. Pour qu'il y ait production du sens, il faut qu'un contexte s'accorde à une subjectivité. Tout contenu est crédité d'un sens, pourvu que le contexte dans lequel il est émis suffise à garantir le rapport aux signifiés. C'est précisément cet encadrement conceptuel qui devra délimiter l'espace des possibles. Mais il y a toujours le sens pour moi et le sens pour l'autre.

Indépendamment de ces données, on ne peut nullement prévoir de manière normative ce qui se passera. On peut seulement prescrire la nécessité du questionnement qui conduit à une humilité lucide et favorise la prise de conscience des différentes modalités du texte d'écran et de leurs limites.

\section{Le contexte numérique : le support idéal pour les fragments?}

Cette approche de la création du sens par le lecteur nous oriente vers la problématique de l'édition de fragments, qui historiquement éclaire les enjeux liés au support. La quête du philosophe Wittgenstein, notamment, vers un livre qui soit un livre et non simplement un album de pensées, est révélatrice d’une tension singulière, comme le relate l'auteur dans sa préface des Investigations philosophiques ${ }^{10}$. Le fragment, cette unité discursive autonome par rapport à la

9. J.-P. Balpe, Contextes de l'art numérique, Hermès, 2000, p. I 2.

Io. L. Wittgenstein, Tractatus logico-philosophicus suivi de Investigations philosophiques, Gallimard, i 96 I, p. i I I. 
linéarité de l'écriture et de la lecture mais dont l'autonomie n'est pas incompatible avec l'unité du livre ${ }^{11}$, met singulièrement en lumière la chaîne d'opérations techniques et intellectuelles qui préside à la mise en texte. En effet, l'histoire de l'édition de textes fragmentaires s'accompagne de controverses éditoriales qui tiennent à l'ordonnancement des fragments. Pour se rappeler ces enjeux, il suffit de relire les préfaces des éditions successives des Pensées de Pascal, qui questionnent l'ordre à établir. La tension entre un ordre de fait et le maintien de l'absence de tout ordre de droit éclaire notamment l'importance du sens en contexte.

L'édition numérique interroge à nouveau ce débat ancien. Tout porte à croire que l'hypertexte, comme technologie intellectuelle et énonciative qui rompt avec la linéarité du discours, soit le dispositif idéal pour accueillir les textes fragmentaires. C'est notamment le sens de l'initiative d'une maquette d'édition hypertextuelle du Tractatus de Wittgenstein par Enrico Bartollucci ${ }^{12}$ qui, à partir d'une démonstration du caractère hypertextuel de cette œuvre fragmentaire, a tenté d'en rendre explicite la structure arborescente en s'appuyant sur le système de numérotation. L'idée était de proposer une lecture hypertextuelle de l'œuvre plus appropriée que la lecture de l'édition papier. Le lecteur pourrait visualiser les liens logiques, ce qui favoriserait des échos entre les questions abordées par le philosophe. Mais cela fait question, dans la mesure où le lien hypertextuel supplée à ce qui était, dans l'ordre du livre, laissé à l'appréciation du lecteur, à savoir l'élaboration des liens. Les fragments, comme dispositifs à trous, instaurent des échos. Or l'hypertexte est à mi-chemin entre le fragment et la linéarité, structure reliée déconstruite et recomposée par le lecteur, à qui il appartient de s'approprier le texte.

Pour conclure sur ces éléments de réflexion, la dimension fonctionnelle de l'outil numérique ne peut suffire : elle doit intégrer la complexité de la mise en texte d'un contenu numérique, qui transforme. Ce que nous apprennent les apports méthodologiques de la conception, c'est que ce passage des modalités de production du sens à la création de sens doit être pensé en fonction de l'expérience du lecteur. Le «faire technique» tend à éluder l'essentiel : c'est-à-dire la question de l'usage. Pour répondre à la question «convient-il ou ne convient-il pas d'adopter la solution numérique?» posée par ce colloque, soulignons la nécessité d'une réflexion sur la forme, mais plus encore sur la conscience critique de ce qui se joue dans le contexte numérique. Si le dispositif interactif tend à être

I I. C. Angé, «La question du sens : écrire \& lire le fragment. Du texte à l'hypertexte», thèse de Doctorat, Université Paris XIII, 2005.

I 2. E. Bartolluci, «Technologie de l'hypertexte et philosophie hypertextuelle», Le Philosophoire $\mathrm{n}^{\circ} 4,1998$. 


\section{Recherches \& Travaux $-\mathrm{n}^{\circ} 72$}

formaté, l'appropriation, elle, ne peut l'être. La rencontre n'est pas déterminée $a$ priori; tenter de la modéliser ne se réduit qu'à ouvrir un espace de possibles : celui du texte et de son lecteur. Il est parfois plus facile de faire fonctionner des objets que de les interroger. De l'accès à l'information, de la mise en ligne à son appropriation par un lecteur-acteur, il n'y a pas qu'un pas. Il y a bien plus. La publication numérique exige d'être guidée par une réflexion soutenue sur ce qui fait sens, dans la mesure où éditer est un acte communicationnel. 